\title{
Pseudomonas aeruginosa outcompetes other bacteria in the manifestation and maintenance of a biofilm in polyvinylchloride tubing as used in dental devices
}

\author{
Christoph Gert Ammann ${ }^{1}$ - Markus Nagl ${ }^{2}$ Michael Nogler ${ }^{1}$. \\ Débora Cristina Coraça-Huber ${ }^{1}$ (D)
}

Received: 1 February 2016 / Revised: 23 February 2016 / Accepted: 1 March 2016 / Published online: 15 March 2016

(C) The Author(s) 2016. This article is published with open access at Springerlink.com

\begin{abstract}
In a PVC tube as a model system for dental devices, Pseudomonas aeruginosa outcompetes Staphylococcus aureus and Klebsiella pneumoniae for the biofilm formation. $P$. aeruginosa has advantage over the other strains due to higher tolerance for low-nutrient situations or direct killing by the production of soluble factors like pyocyanin.
\end{abstract}

Keywords Bacterial biofilm $\cdot$ PVC tubing $\cdot$ Dental device $\cdot$ Co-culture

\section{Findings}

Polyvinylchloride (PVC) tubes are widely used in medical and dental devices. These tubes can easily come in contact with human skin and mucosa during odontology procedures and can be contaminated with the following bacteria that play a role in human medicine.

Pseudomonas aeruginosa is a Gram-negative rod-shaped bacterium found in moist to wet habitats. These include human mucosal surfaces, e.g., the nasopharynx (Fothergill et al. 2014), and surfaces in tap water lines (Rozej et al. 2015). Klebsiella pneumoniae is a Gram-negative

Communicated by Erko Stackebrandt.

Débora Cristina Coraça-Huber

debora.coraca-huber@i-med.ac.at

1 Experimental Orthopaedics, Medical University of Innsbruck, Innrain 36 - 1. Floor, 6020 Innsbruck, Austria

2 Division of Hygiene and Medical Microbiology, Department of Hygiene, Microbiology and Social Medicine, Medical University of Innsbruck, Schöpfstrasse. 41, 6020 Innsbruck, Austria rod-shaped bacterium that can colonize mouth mucosal tissue (Bagley 1985) and cause pneumonia (Podschun and Ullmann 1998). Staphylococcus aureus is a Gram-positive bacterium that typically colonizes skin. Approximately $20 \%$ of all human beings are long-term carriers of $S$. aureus in nose mucosa (Kluytmans et al. 1997).

Once in contact with the moist surface of a tube lumen, bacteria can adhere to the material and start biofilm formation. Biofilms are formed in several stages during propagation of bacteria after adherence (Stoodley et al. 2002). Once a sufficient number of bacteria are reached, the biofilm matures, ultimately establishing an extracellular matrix (ECM). The ECM contains water, polyglycans, proteins, and nucleotides (Branda et al. 2005). Bacteria in the biofilm use these stored materials in times of malnutrition from the exterior.

We here investigate the growth of three bacterial strains which are typical components of human flora. These can transfer from human to a dental device and subsequently grow in the lumen of the PVC tubing. We sought to determine the growth pattern and dynamics of $S$. aureus and $K$. pneumoniae in co-culture with $P$. aeruginosa, a well-known contaminant of water systems.

Pseudomonas aeruginosa ATCC 27853, S. aureus ATCC 25923, and K. pneumoniae (clinical isolate) single colonies from Mueller-Hinton (MH) agar were grown separately overnight at $37{ }^{\circ} \mathrm{C}$ in $\mathrm{MH}$ broth to $2-5 \times 10^{9}$ colony-forming units $(\mathrm{cfu}) / \mathrm{mL}$. Subsequently, $P$. aeruginosa was mixed with $S$. aureus or K. pneumoniae and diluted in MH broth to approximately $1 \times 10^{5} \mathrm{cfu} / \mathrm{ml}$ for each strain. One tube system each was filled with $P$. aeruginosa plus $S$. aureus or $P$. aeruginosa plus $K$. pneumoniae. We here sought to speed up the process of initial biofilm formation by providing ample nutrients (MH broth) for $72 \mathrm{~h}$. After this period, we provided tap water for the system and followed the bacterial 
count of the formed biofilms for 5-8 weeks. To imitate a dental device system, we cultivated the biofilm at room temperature and provided flow of water by a peristaltic pump.

We used scanning electron microscopy to visualize the biofilms on the tube lumen and collect data on their spatial distribution at the end of the incubation period. After fixation and dehydration, samples were investigated in a scanning electron microscope (Jeol 6010, Eching, Germany) at $5 \mathrm{kV}$ acceleration voltage using a spot size of 40 or 50 , respectively. We used the REF detector setting in which data are collected through the secondary electron detector without using the suction current to attract secondary electrons. We found that biofilms are clustered in small groups of high bacterial counts instead of forming a single low-density biofilm spread over the entire tube lumen (Fig. 1a, b). Similar results have been observed with confocal microscopy using ground water and no specific bacterial input (Martiny et al. 2003).

The single biofilms showed a thick ECM spreading over the biofilm core, while single bacteria could still be visualized at the edges of the individual biofilms. A representative image is shown in Fig. 1b. At weekly intervals, a sample of the PVC tube was cut and stained with $0.5 \%$ crystal violet for $5 \mathrm{~min}$ for macroscopic evaluation of the biofilm growth. We observed a gradual covering of the lumen along the time beginning with isolated clumps. Additionally, we weekly cut a $10-\mathrm{cm}$ piece of tubing. $3 \mathrm{~cm}$ were cut from the non-pump side, washed extensively, and placed in $6 \mathrm{ml}$ of $\mathrm{MH}$ medium. The biofilm was then detached from the tube by ultrasound $(41 \mathrm{kHz}$, Bandelin Bactosonic, Berlin, Germany) for $1 \mathrm{~min}$. Bacterial counts were determined after transferring $100 \mu \mathrm{l}$ of the solution to a $\mathrm{MH}$ agar plate leading to a limit of detection of $10 \mathrm{cfu} / \mathrm{ml}$. At end, we calculated how many bacteria were attached to the tube lumen per square centimeter. Figure 2 shows the determined values for both experimental settings combined.

Pseudomonas aeruginosa proved to be the predominant bacterial strain overgrowing both $S$. aureus and K. pneumoniae. All strains were initially detected at a similar level of approximately $10^{5} \mathrm{cfu} / \mathrm{cm}^{2}$. After only 2 weeks, $S$. aureus had vanished from the system, while $P$. aeruginosa showed still increasing colony counts. K. pneumoniae, after slightly increasing from 5.01 to $5.83-5.89 \log _{10} \mathrm{cfu} / \mathrm{cm}^{2}$ during the initial 2 weeks, decreased rapidly in the third week and was undetectable at any time point in the following weeks. The overgrowth by $P$. aeruginosa may either be an indirect effect of this species being better adapted to low-nutrient situations, or it may reflect a direct killing of the partners in co-culture by secreted toxins like pyocyanin (Voggu et al. 2006). In contrast to the vanishing of $S$. aureus or $K$. pneumoniae, the cfu counts of $P$. aeruginosa plateaued rather stably at approximately $200 \mathrm{cfu} / \mathrm{cm}^{2}$ of tube lumen from week six on. This is in agreement with early reports
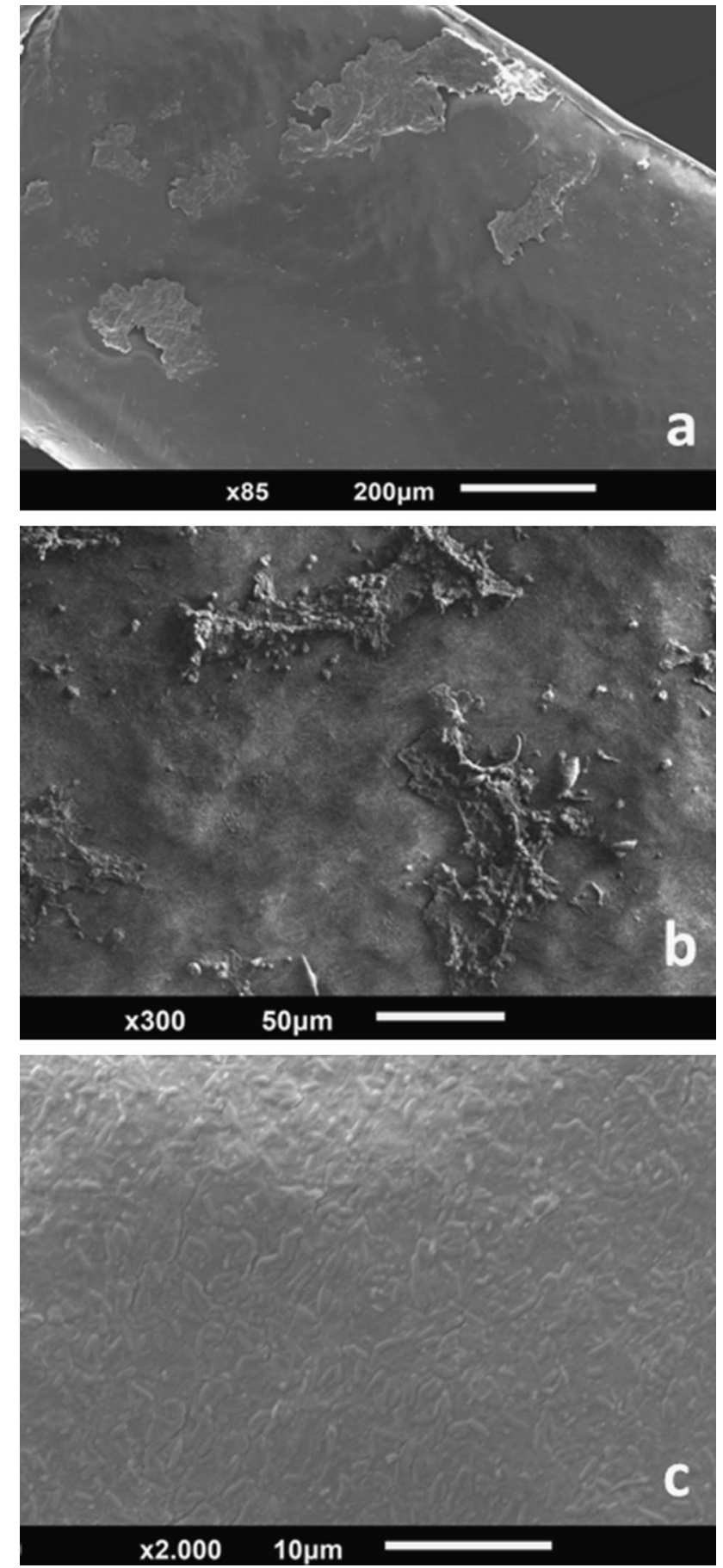

Fig. 1 Scanning electron microscopy image of bacterial biofilms spread over the PVC tube lumen. a Small areas of biofilms spread on the PVC tube lumen; b Staphylococcus aureus biofilm on PVC lumen; c PVC lumen populated by Pseudomonas aeruginosa biofilm

showing that in dental unit water lines shedding of $10^{2}$ to $10^{6} \mathrm{cfu} / \mathrm{ml}$ can occur (Gross et al. 1976; Furuhashi and Miyamae 1985).

Our data show that biofilms can be formed and maintained in tap water on the lumen of PVC tubes using the 


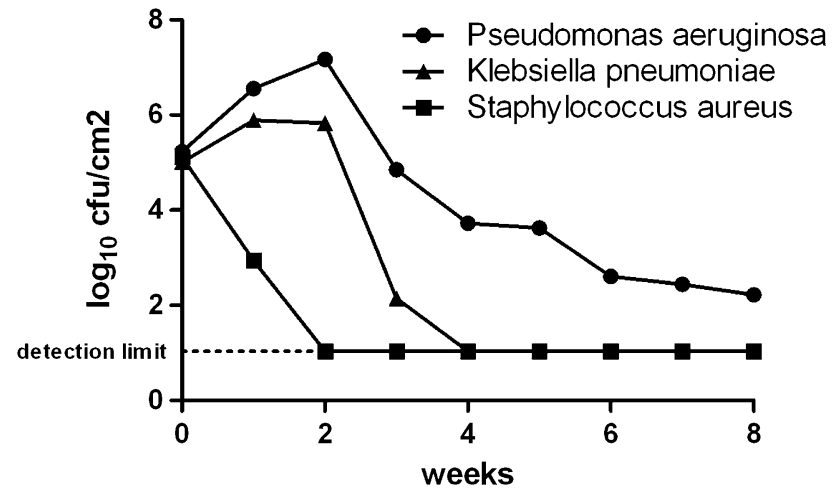

Fig. 2 Bacterial counts per square centimeter of tubing; mean values of $n=2$ for $P$. aeruginosa (filled circle), single values for K. pneumoniae (filled triangle) and $S$. aureus (filled square)

method developed here. Pseudomonas outcompeted the respective partners in co-cultures and established long-lived biofilms in water according to our experiments. The longtime incubation of $P$. aeruginosa with water has recently been shown to alter the phenotype significantly (Mendis et al. 2014). Our future experiments will focus on the genotypic and resulting phenotypic changes in long-term dental unit biofilms as well as the sterilization of biofilms with novel agents.

Acknowledgments We thank Sebastian Geiger and Bruno Prgenzer from Metasys Medizintechnik GMBH for providing the PVC tubing and the lively discussion. We also thank Andrea Windisch for her excellent technical help during the experiments.

Open Access This article is distributed under the terms of the Creative Commons Attribution 4.0 International License (http://creativecommons.org/licenses/by/4.0/), which permits unrestricted use, distribution, and reproduction in any medium, provided you give appropriate credit to the original author(s) and the source, provide a link to the Creative Commons license, and indicate if changes were made.

\section{References}

Bagley ST (1985) Habitat association of Klebsiella species. Infect Control 6(2):52-58

Branda SS, Vik S, Friedman L, Kolter R (2005) Biofilms: the matrix revisited. Trends Microbiol 13(1):20-26. doi:10.1016/j. tim.2004.11.006

Fothergill JL, Neill DR, Loman N, Winstanley C, Kadioglu A (2014) Pseudomonas aeruginosa adaptation in the nasopharyngeal reservoir leads to migration and persistence in the lungs. Nat Commun 5:4780. doi: $10.1038 /$ ncomms5780

Furuhashi M, Miyamae T (1985) Prevention of bacterial contamination of water in dental units. J Hosp Infect 6(1):81-88

Gross A, Devine MJ, Cutright DE (1976) Microbial contamination of dental units and ultrasonic scalers. J Periodontol 47(11):670673. doi:10.1902/jop.1976.47.11.670

Kluytmans J, van Belkum A, Verbrugh H (1997) Nasal carriage of Staphylococcus aureus: epidemiology, underlying mechanisms, and associated risks. Clin Microbiol Rev 10(3):505-520

Martiny AC, Jørgensen TM, Albrechtsen H-J, Arvin E, Molin S (2003) Long-term succession of structure and diversity of a biofilm formed in a model drinking water distribution system. Appl Environ Microbiol 69(11):6899-6907. doi:10.1128/ AEM.69.11.6899-6907.2003

Mendis N, Lin YR, Faucher SP (2014) Comparison of virulence properties of Pseudomonas aeruginosa exposed to water and grown in rich broth. Can J Microbiol 60(11):777-781. doi:10.1139/ cjm-2014-0519

Podschun R, Ullmann U (1998) Klebsiella spp. as nosocomial pathogens: epidemiology, taxonomy, typing methods, and pathogenicity factors. Clin Microbiol Rev 11(4):589-603

Rozej A, Cydzik-Kwiatkowska A, Kowalska B, Kowalski D (2015) Structure and microbial diversity of biofilms on different pipe materials of a model drinking water distribution systems. World J Microbiol Biotechnol 31(1):37-47. doi:10.1007/ s11274-014-1761-6

Stoodley P, Sauer K, Davies DG, Costerton JW (2002) Biofilms as complex differentiated communities. Annu Rev Microbiol 56:187-209. doi:10.1146/annurev.micro.56.012302.160705

Voggu L, Schlag S, Biswas R, Rosenstein R, Rausch C, Götz F (2006) Microevolution of cytochrome bd oxidase in staphylococci and its implication in resistance to respiratory toxins released by pseudomonas. J Bacteriol 188(23):8079-8086. doi:10.1128/ JB.00858-06 